(5) $\left\{\left\{\left(a_{2} b_{3}-a_{3} b_{2}\right) x_{1}+\left(a_{3} b_{1}-a_{1} b_{3}\right) x_{2}+\left(a_{1} b_{2}-a_{2} b_{1}\right) x_{3}\right\}\right.$ $\times\left\{s_{1} x_{2} x_{3}\left(x_{2}-x_{3}\right)+s_{1} x_{3} x_{1}\left(x_{3}-x_{1}\right)\right.$

$$
\left.+s_{3} x_{1} x_{2}\left(x_{1}-x_{2}\right)\right\}=0 .
$$

Clearly, the quartic degenerates into the $S$-line of the pencil of cubics, and into a cubic having $\left(s_{1}, s_{2}, s_{3}\right)$ as the $S$-point. Hence the

Theorem: Any cubic of the net with a given S-point may be generated by any pencil of cubics within the net, not containing the given cubic, and the projective pencil of lines joining the Spoint of the given cubic to the $S$-points of the cubics of the pencil.

Consider next two pencils of cubics $C_{\lambda}$ with the $S$-line $l$, and $C_{\mu}$ with the $S$-line $m$, and a point $S$, not on $l$ or $m$. Draw any line $g$ through $S$, cutting $l$ and $m$ in $S_{\lambda}$ and $S_{\mu}$, and construct the cubics $C_{\lambda}$ and $C_{\mu}$ having $S_{\lambda}$ and $S_{\mu}$ as $S$-points.

They both pass through the two fixed points $P$ and $P^{\prime}$ on $g$ corresponding to each other in the Steinerian transformation. But $P$ and $P^{\prime}$ also lie on the cubic $C_{S}$ associated with $S$ as an $S$-point. For a variable $g$ through $S, S_{\lambda}$ and $S_{\mu}$ describe two perspective point sets on $l$ and $m$ which are projective with the pencils of cubics $C_{\lambda}$ and $C_{\mu}$. These pencils are therefore themselves projective, and generate the cubic $C_{S}$. Hence the

TheOREM: Every cubic of the net associated with a Steinerian transformation may be generated in an infinite number of ways by projective pencils of cubics of the same net.

UNIVERSITY OF ILLINOIS.

\title{
SOME THEOREMS OF COMPARISON AND OSCILLATION.
}

BY PROFESSOR TOMLINSON FORT.

(Read before the American Mathematical Society September 4, 1917.)

Theorem I: Given

$$
\frac{d}{d x}\left(K_{1}(x) y_{1}{ }^{\prime}\right)+G_{1}(x) y_{1}=0
$$

and

$$
\frac{d}{d x}\left(K_{2}(x) y_{2}{ }^{\prime}\right)+G_{2}(x) y_{2}=0,
$$


where $K_{1}, K_{2}, G_{1}$ and $G_{2}$ are real and absolutely integrable in the Riemann sense over the interval $a \leqq x \leqq b$, and where $0<K_{2} \leqq K_{1}$ and $0<G_{1}<G_{2}$.

Let $y_{1}$ and $y_{2}$ be particular, not identically vanishing, solutions of (1) and (2) respectively such that either

Case I

$$
y_{1}(a)=0
$$

or

Case II

$$
\frac{K_{1}(a) y_{1}^{\prime}(a)}{y_{1}(a)} \geqq \frac{K_{2}(a) y_{2}^{\prime}(a)}{y_{2}(a)} .
$$

Let $V_{i}(x)=\alpha_{1}(x) K_{i}(x) y_{i}{ }^{\prime}(x)+\alpha_{2}(x) y_{i}(x), i=1,2$, where $\alpha_{1}(x)$ and $\alpha_{2}(x)$ are real functions defined and continuous when $a \leqq x \leqq b$ and such that $\alpha_{1}(x) \neq 0$ at any point and $\alpha_{2}(x) / \alpha_{1}(x)$ never decreases as $x$ increases. Assume that $y_{1}(a) \geqq 0, y_{2}(a) \geqq 0$ and $V_{1}(a) \cdot V_{2}(a) \geqq 0$ and that, if $V_{2}(a)=0, V_{1}(a)=0$ also.

If $V_{1}(x)$ has a root at a point $c$, where $a<c \leqq b$, then $V_{2}(x)$ has a root at a point $d$, where $a<d<c$.

Without loss of generality we assume $\alpha_{1}>0$. If $\alpha_{1}<0$ we simply change the signs of both $\alpha_{1}$ and $\alpha_{2}$.

Case I. Assume $y_{1}{ }^{\prime}(a)>0$ and, if $y_{2}(a)=0, y_{2}{ }^{\prime}(a)>0$ also, in which again there is no loss of generality. Let $l=a$ $+\delta$ where $\delta>0$ is very small. Then

$$
\begin{aligned}
\left.K_{1} y_{1}{ }^{\prime} y_{2}-K_{2} y_{2}{ }^{\prime} y_{1}\right]_{a}^{l}=\int_{a}^{l}\left(G_{2}-G_{1}\right) y_{1} y_{2} d x & \\
& +\int_{a}^{l}\left(K_{1}-K_{2}\right) y_{1}{ }^{\prime} y_{2}{ }^{\prime} d x .
\end{aligned}
$$

In case $y_{2}(a)=0$ and $\delta$ is sufficiently small, $y_{1} y_{2}>0$ when $a<x \leqq l$ and $y_{1}^{\prime} y_{2}^{\prime}>0$ when $a \leqq x \leqq l$. Consequently

$$
\frac{K_{1}(l) y_{1}^{\prime}(l)}{y_{1}(l)}>\frac{K_{2}(l) y_{2}{ }^{\prime}(l)}{y_{2}(l)} \text {. }
$$

If $y_{2}(a) \neq 0$ this result is immediate from the continuity of $y_{1}, y_{1}{ }^{\prime}, y_{2}$ and $y_{2}{ }^{\prime}$. Replace the point $a$ by the point $l$ and Case I reduces to Case II.

Case II. Assume that $V_{2}(x)$ does not have a root on the interval $a<x<c$ and assume that $c$ is the first root of $V_{1}(x)$ greater than $a$. Choose $y_{1}(a)=y_{2}(a)$.

(a) Suppose $V_{2}(a)>0$ and consequently $V_{1}(a)>0$. 


$$
\begin{aligned}
\left.y_{1}{ }^{2}\left(\frac{K_{1} y_{1}{ }^{\prime}}{y_{1}}-\frac{K_{2} y_{2}{ }^{\prime}}{y_{2}}\right)\right]_{a}^{c}=\int_{a}^{c}\left(G_{2}-G_{1}\right) y_{2}{ }^{2} d x \\
\quad+\int_{a}^{o}\left(K_{1}-K_{2}\right){y_{2}}^{\prime 2} d x+\int_{a}^{c} K_{2}\left(\frac{y_{1}{ }^{\prime} y_{2}-y_{1} y_{2}{ }^{\prime}}{y_{2}}\right)^{2} d x .^{*}
\end{aligned}
$$

$y_{1}$ and $y_{2}$ are both positive over the interval of integration since

$$
\frac{K_{1}(x) y_{1}{ }^{\prime}(x)}{y_{1}(x)}
$$

becomes negatively infinite as $x$ approaches $c$, and similarly

$$
\frac{K_{2}(x) y_{2}{ }^{\prime}(x)}{y_{2}(x)}
$$

becomes negatively infinite as $x$ approaches the first root of $y_{2}$ greater than $a$. Consequently

$$
K_{1}(c) y_{1}{ }^{\prime}(c) y_{2}(c)-K_{2}(c) y_{2}{ }^{\prime}(c) y_{1}(c)>0 .
$$

Combining this with $V_{1}(c)=0$, we get $y_{1}(c) V_{2}(c)<0$, which is impossible.

(b) $V_{2}(a) \leqq 0$ and $V_{1}(a) \leqq 0$.

$$
\frac{K_{i}(x) y_{i}{ }^{\prime}(x)}{y_{i}(x)}
$$$$
(i=1,2)
$$

decreases as $x$ increases, as is proved by differentiation. Consequently $y_{i}(x)=0$ to the right of $a$ necessarily before $V_{i}(x)$ $=0$. Suppose $y_{1}=0$ at $g$, where $g<c$; then, as is well known, $y_{2}=0$ at $h$ where $h<g$. There is only one such point less than $c$, for by hypothesis $V_{2}(x)$ has no root when $a<x<c$. Take a point $l=g+\delta$, where $\delta$ is small. At this point, with $\delta$ small enough, we have the same situation as at $a$ under $(a)$ if $y_{2}$ is replaced by $y_{2}$ multiplied by such a constant as to cause $y_{2}(l)$ to equal $y_{1}(l)$ and then $y_{1}$ and $y_{2}$ replaced by $-y_{1}$ and $-y_{2}$ respectively.

Theorem II. Let $y_{1}$ and $y_{2}$ be any two not identically vanishing solutions of (1) and (2) respectively and let

$$
V_{i}(x)=\alpha_{1}(x) K_{i}(x) y_{i}{ }^{\prime}(x)+\alpha_{2}(x) y_{i}(x) \quad(i=1,2) .
$$

Between any two successive roots of $V_{1}(x)$ on the interval $a \leqq x \leqq b$ lies at least one root of $V_{2}(x)$.

\footnotetext{
*This is a well known identity. It can easily be verified.
} 
Suppose two successive roots of $V_{1}(x)$ at $c_{1}$ and $c_{2}$ respectively. Without loss of generality we assume $y_{1}\left(c_{1}\right)>0$ and $y_{2}\left(c_{1}\right) \geqq 0 ; \quad$ if $y_{2}\left(c_{1}\right)=0, \quad y_{2}{ }^{\prime}\left(c_{1}\right)>0$. Since in addition $K_{1}(x) y_{1}^{\prime}(x) / y_{1}(x)$ decreases in $x$ and $\alpha_{1}>0, V_{1}(x)<0$ when $c_{1}<x<c_{2}$.

Case I. $V_{2}(x)<0$ when $c_{1}<x<c_{2}$. Conditions at $a$ of Theorem I, Case II $b$, are fulfilled at $c_{1}$.

Case II. $V_{2}(x)>0$ when $c_{1}<x<c_{2}, y_{1}=0$ at $g$, where $c_{1}<g<c_{2}$, since $K_{1}(x) y_{1}{ }^{\prime}(x) / y_{1}(x)$ always decreases. At $g$ we have the conditions of Theorem I, Case I, $y_{1}$ being replaced by $-y_{1}$.

Suppose next that we have the equation

$$
\frac{d}{d x}\left(K(x, \lambda) y^{\prime}\right)+G(x, \lambda) y(x)=0,
$$

where $K(x, \lambda)$ and $G(x, \lambda)$ are real and continuous in $\lambda$, $-\infty<\lambda<\infty$, for any $x$, and with respect to $x$ absolutely integrable in the Riemann sense from $a$ to $b$, and where $K>0$ never decreases as $\lambda$ increases, and $G$ actually increases from values less than or equal to zero to values as large as desired and is such that, for a particular $\lambda, G \equiv 0$ in $x$ or does not vanish at all.

Consider (3) subject to the boundary conditions

$$
\begin{aligned}
& \beta_{1} K(a, \lambda) y^{\prime}(a)+\beta_{2} y(a)=0, \\
& \alpha_{1} K(b, \lambda) y^{\prime}(b)+\alpha_{2} y(b)=0,
\end{aligned}
$$

where $\alpha_{1}, \alpha_{2}, \beta_{1}$ and $\beta_{2}$ are real constants, $\alpha_{1}>0$ and $\beta_{1}$ and $\beta_{2}$ are not both zero.

Let $V(x)=\alpha_{1} K(x, \lambda) y^{\prime}(x)+\alpha_{2} y(x)$. It is to kept in mind that from now on $\alpha_{1}$ and $\alpha_{2}$ are constants.

Define $K(x, \lambda)$ and $G(x, \lambda)$, when $x>b$, thus: $K(x, \lambda)$ $=K(x, b)$ and $G(x, \lambda)=G(x, b)$.

Let $y$, not identically equal to zero, satisfy the first of equations (4). Then notice that $V$ and $V^{\prime}$ are continuous in $\lambda$ and that they do not simultaneously vanish when $G \geqq 0$.

Moreover when $G \equiv 0$ and $V(x) \neq 0, V(x)$ has at most one root on the interval $a \leqq x \leqq b$, as is readily proved by solving the equation. Consequently in this case when $G>0$ but sufficiently small, $V(x)$ has only one root on the interval $a \leqq x \leqq b$. $V \equiv 0$ is only possible when $V=0$ reduces to $y^{\prime}=0$. For values of $G>0$ but sufficiently small in this instance, $V \neq 0$ at all on the interval $a<x \leqq b$, since $K(x, \lambda) y^{\prime}(x) / y(x)$ 
decreases as $\lambda$ increases. Moreover when $\lambda$ is sufficiently large, as is well known, $y$ and consequently $V$ have as many roots as desired when $a<x<b$. Using these facts and Theorems I and II we now readily prove the following:

Theorem III. There exist unique values, $\lambda_{1}<\lambda_{2}<\lambda_{3}$ $<\cdots$, such that when $\lambda=\lambda_{j}, j=1,2,3, \cdots$, a solution $y$ of (3) exists satisfying (4) such that $V(x)$ has exactly $j$ roots on the interval $a<x<b$.

We can extend this to $j=0$ if, when $G=0, V$ has no root on the interval $a<x<b$.

The University of Alabama, September, 1917.

\section{NOTE ON INFINITE SYSTEMS OF LINEAR EQUATIONS.}

BY DR. W. L. HART.

(Read before the American Mathematical Society April 28, 1917.)

IN considering infinite systems of linear equations

$$
\sum_{j=1}^{\infty} a_{i j} x_{j}=x_{i}{ }^{\prime} \quad(i=1,2, \cdots),
$$

particular interest is attached to those whose solutions preserve the properties of the solutions of a set of $n$ linear equations in $n$ variables. It is known* that the system (1) possesses this property if $a_{i j}=d_{i j}-b_{i j}\left(d_{i i}=1 ; d_{i j}=0, i \neq j\right)$, where the infinite matrix $B=\left(b_{i j}\right)_{i, j=1,2}, \ldots$ is completely continuous. $\dagger$ The discussion of Riess deals only with the special case $a_{i j}=d_{i j}-b_{i j}$, but it is easily found that his proof holds for the more general case stated below. The proof of the theorem of this note is not given since it differs only in minor details from the proof of the theorem given by Riess.

It will be said that a matrix $A_{1}$ is a sub-matrix of the matrix

* Cf. F. Riess, Equations Linéaires, p. 94.

$\dagger$ Cf. F. Riess, loc. cit. 